\title{
Future Trading in India and Commodity Price Risk Management: A Pragmatic Study
}

\author{
Nirmala K. Reddy*, B. M. Chandra Shekar and R. Munilakshmi
}

Department of Commerce, Bangalore University, India

\begin{abstract}
Commodity future markets in India are experiencing unparalleled growth and have attained critical economic significance in the last one decade. On the other hand, instability in commodity prices is becoming an issue of great concern not only for India, but all over the world impacting income, economic growth and a poor adversely. Ever-increasing demand and supply side constraints are adding to the upsurge in prices of metal and agricultural commodities, affecting manufacturers and consumers at the same time. Moreover, farmer participation in the market has been very poor. So the price risk management in commodity is not a cliché but a necessity for the development of future market. In an agriculture based economy like India, commodity derivatives are expected to play a pivotal role in the process of price discovery and risk management. The price discovery in futures markets would not be effective unless spot markets are regulated and integrated.

The present paper aims to analyse the performance of futures trading in improvising commodity price risk management in India. The study employs co-integration technique to study the existence of long-term relationship between the spot and future prices of agricultural and metal commodities traded in Indian commodity exchanges. The study also explores the volatility aspect in spot and future prices to test the informational efficiency of the contracts and comment on their suitability for hedging activities. Based on the results, propositions would be made on the nature of speculative conditions and offer suggestions for improvement futures trading in commodities.
\end{abstract}

Keywords: Co-integration, Liquidity, Price Risk Management

\section{Introduction}

Derivative markets are price discovery and risk management institutions. In derivative markets, the competing expectations of traders interact to 'discover' prices. In doing so, they reflect a broad range of information that exists on upcoming market conditions. Derivative markets are actually designed as vehicles for establishing future prices and managing risk. Commodity derivatives have a crucial role to play in the price risk management process especially in any agriculture dominated economy like India and instability in commodities continue to be the major concern of the producers as well as the consumers. Exposure of farmers to the price volatilities of the market make it very risky for them to invest in otherwise profitable activities. There are various ways to cope with this problem. Apart from increasing the stability of the market, various actors in the farm sector can better manage their activities in an environment of unstable prices through derivative markets. These markets serve as a risk-shifting function, and can be used to lock-in prices instead of relying on uncertain price developments.

With gradual realisation and acceptance by the country's economic stakeholders that both global 
availability and prices of international commodities have to be factored in, to take the necessary production and other commercial decisions, there is also an implicit recognition of the necessity of shielding their commercial operations from risks posed by commodity price volatility at the international levels. There has been overwhelming recognition by Indian companies of the risks arising out of commodity price fluctuations and, the importance of hedging of inputs at pre-fixed prices as a risk-mitigating strategy.

Commodity markets in India are experiencing revolutionary growth in commodity derivatives trading. Since the removal of restriction on trade in 2002, the commodity markets are growing fiercely. Now we have 6 national level electronic exchanges and 16 regional exchanges for trading commodity derivatives. A total of 109 commodities have been allowed for trading. A brief account of present trends in commodity trading in India is presented below.

In the year 2011-12, the total market size of the commodity futures market in India was about Rs. 11,81,261 billion with a Compound Annual Growth Rate (CAGR) of around $40 \%$ since its renewal in 2003. In terms of trade volume, energy leads other commodities followed by agricultural commodities and industrial metals. On the other hand, Precious Metals (Bullions), which constitute less than $1 \%$ of the trade volume accounts for $56 \%$ of the total, traded value in 2011-12, followed by Industrial Metals (16\%), Agriculture (12\%) and Energy (16\%) Commodities (Table 1).

Between 2009-10 and 2011-12, futures trading was regulated in 109 commodities at 21 recognized exchanges and the Bullions (precious metals) continued their leadership in futures trading and the same was more evident during 2011-12. The trade in industrial metals, agricultural commodities and energy has seen marginal growth (Chart 1).

In India, national level exchanges dominate the commodity trading scene both in value and volume. These are basically traded on electronic platform with option round the clock for information sharing and transaction monitoring. More than $99 \%$ of these trading-by-value
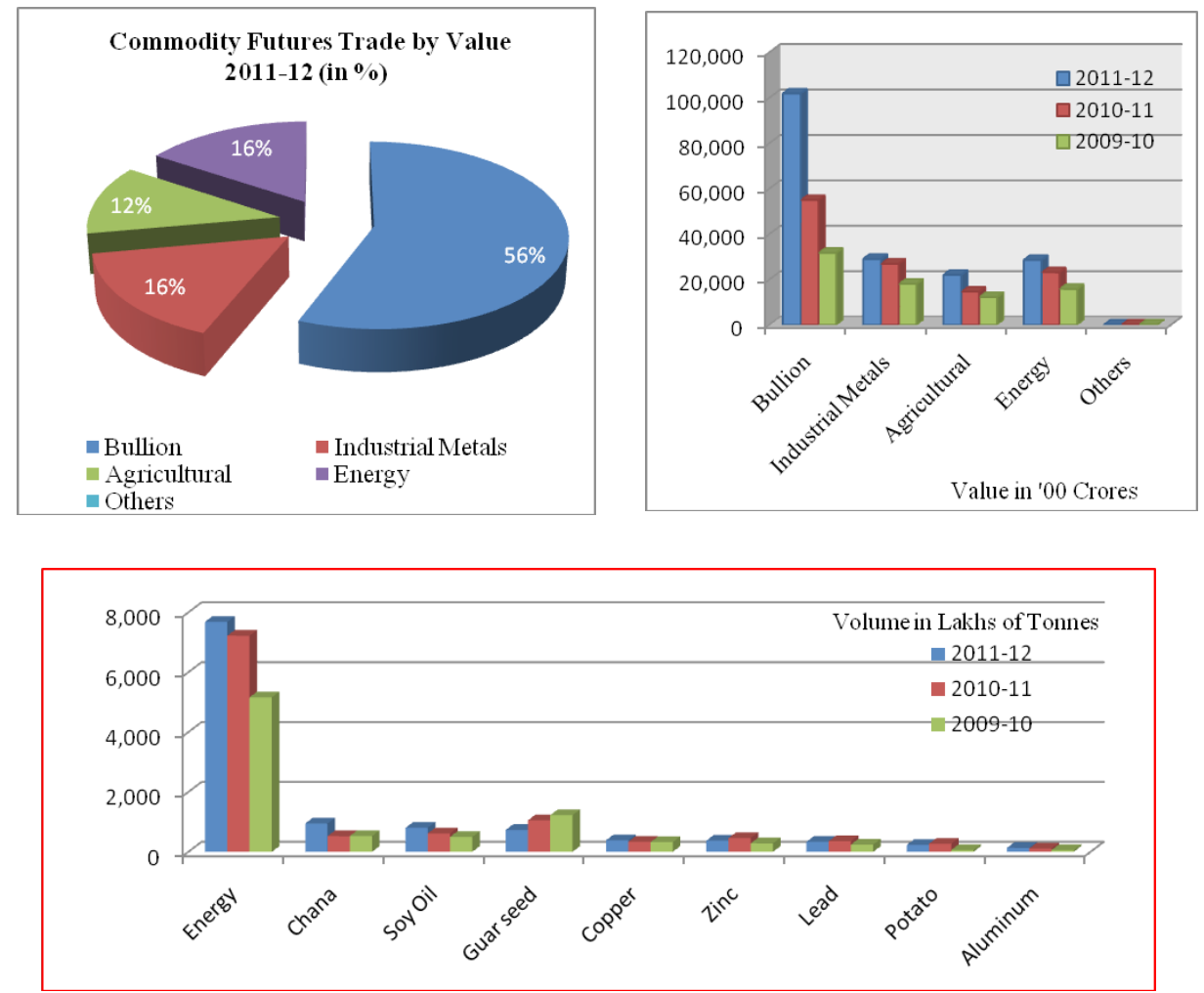

Chart 1. Commodities Futures by cateogory and trade value. 
Table 1. Growth in commodity futures in India by trade volume and value

\begin{tabular}{|c|c|c|c|c|c|c|c|}
\hline \multicolumn{8}{|c|}{ Volume of trading In lakh tonnes/value in crore of rupees } \\
\hline \multirow[t]{2}{*}{ SI. No. } & \multirow[t]{2}{*}{ Name the Commodity } & \multicolumn{2}{|c|}{ 2011-12 } & \multicolumn{2}{|c|}{ 2010-11 } & \multicolumn{2}{|c|}{ 2009-10 } \\
\hline & & Volume & Value & Volume & Value & Volume & Value \\
\hline A & Bullion & & & & & & \\
\hline $\mathrm{i}$ & Gold & 0 & $4,355,099$ & 0 & $2,700,607$ & 0 & $1,997,801$ \\
\hline ii & Silver & 10 & $5,826,849$ & 7 & $2,793,280$ & 5 & $1,165,729$ \\
\hline iii & Platinum & 0 & 10 & 0 & 5 & 0 & 623 \\
\hline \multicolumn{2}{|c|}{ Total (A) } & 10 & $10,181,957$ & 7 & $5,493,892$ & 5 & $3,164,152$ \\
\hline B & Metals other than Bullion & & & & & & \\
\hline i & Aluminum & 132 & 145,899 & 110 & 114,082 & 57 & 53,904 \\
\hline ii & Copper & 386 & $1,565,984$ & 335 & $1,239,261$ & 326 & 942,590 \\
\hline iii & Lead & 336 & 364,265 & 357 & 366,422 & 245 & 240,269 \\
\hline iv & Nickel & 43 & 427,336 & 45 & 478,789 & 34 & 284,602 \\
\hline $\mathrm{v}$ & Steel & 37 & 11,511 & 87 & 22,759 & 36 & 8,814 \\
\hline vi & Tin & 0 & 3 & 0 & 18 & 0 & 133 \\
\hline vii & Zinc & 373 & 375,693 & 463 & 465,375 & 284 & 271,324 \\
\hline viii & Iron & 81 & 6,031 & 13 & 966 & & \\
\hline \multicolumn{2}{|c|}{ Total (B) } & 1,388 & $2,896,721$ & 1,410 & $2,687,673$ & 982 & $1,801,636$ \\
\hline c & Agricultural Commodities & & & & & & \\
\hline i & Chana/Gram & 948 & 306,412 & 524 & 126,158 & 530 & 127,950 \\
\hline ii & Wheat & 22 & 2,661 & 27 & 3,317 & 32 & 4,015 \\
\hline iii & Maize & 19 & 2,294 & 16 & 1,730 & 8 & 791 \\
\hline iv & Soy Oil & 803 & 538,383 & 617 & 345,286 & 501 & 235,606 \\
\hline v & Mentha Oil & 7 & 101,411 & 6 & 60,527 & 2 & 13,173 \\
\hline vi & Guar seed & 733 & 338,216 & 1,056 & 254,691 & 1,227 & 283,431 \\
\hline vii & Guar Gum & 69 & 100,515 & 83 & 49,943 & 59 & 29,594 \\
\hline viii & Potato & 229 & 14,157 & 269 & 14,428 & 62 & 4,576 \\
\hline ix & Chilli & 14 & 11,611 & 11 & 8,494 & 4 & 1,998 \\
\hline $\mathrm{x}$ & Jeera (Cumin seed) & 37 & 55,983 & 43 & 60,864 & 27 & 33,720 \\
\hline xi & Cardamom & 2 & 16,374 & 1 & 10,882 & 0 & 2,504 \\
\hline xii & Pepper & 25 & 79,519 & 42 & 84,786 & 20 & 27,706 \\
\hline xiii & Rubber & 8 & 16,698 & 12 & 23,847 & 6 & 7,123 \\
\hline xiv & Other Agri. Commodities & 2,026 & 611,915 & 1,461 & 411,436 & 1,514 & 445,763 \\
\hline \multicolumn{2}{|c|}{ Total [C] } & 4,942 & $2,196,150$ & 4,168 & $1,456,390$ & 3,991 & $1,217,949$ \\
\hline D & Energy & 7,686 & $2,851,269$ & 7,220 & $2,310,959$ & 5,163 & $1,577,882$ \\
\hline$E$ & Plastic & 0 & 6 & & & 0 & 0 \\
\hline $\mathrm{F}$ & Other & & 1 & 0 & 29 & 2 & 3,134 \\
\hline Grand T & otal $(A+B+C+D+E+F)$ & 14,026 & $18,126,104$ & 12,806 & $11,948,942$ & 10,143 & $7,764,754$ \\
\hline
\end{tabular}

Source: Compiled from Annual Reports of FMC. 
Table 2. Commodity futures in india by exchange and traded value during 2011-12

\begin{tabular}{lll}
\hline Name of Exchange & Value in Crores & Share of Total \\
\hline MCX & $15,597,095$ & $86.00 \%$ \\
NCDEX & $1,810,210$ & $10.00 \%$ \\
NMCE & 268,351 & $1.00 \%$ \\
ICEX & 258,106 & $1.00 \%$ \\
ACE & 138,655 & $0.70 \%$ \\
Regional Exchanges & 53,687 & $0.30 \%$ \\
Grand Total & $\mathbf{1 8 , 1 2 6 , 1 0 4}$ & $\mathbf{1 0 0} \%$ \\
\hline
\end{tabular}

is accounted for by the five national exchanges namely Multi Commodity Exchange of India Ltd. (MCX), Mumbai; National Multi-Commodity Exchange (NMCE), Mumbai; National Commodity and Deriv-atives Exchange Ltd. (NCDEX), Ahmadabad; Indian Commodity Exchange Ltd. (ICE), New Delhi; and Ace Derivatives and Commodity Exchange Ltd, Mumbai. The lower trading volume and value in the remaining 16 regional exchanges are raising the question as to their survival and the FMC is also considering the proposal to merge these exchanges with other national level exchanges (Table 2, Chart 2).

\section{Literature Review}

By participating in the derivatives markets, producers can offset potential losses in spot markets. A sufficiently large number of studies have been conducted both within and outside India to test the efficiency of commodity derivative markets. Despite presence of a considerable amount of empirical literature using different techniques, there is no general consensus on whether or not the markets are efficient. To quote a few examined the hypothesis that futures prices are unbiased predictors of the subsequent spot prices for the markets of copper, tin, lead and zinc, using daily price data from the LME for the period 1971-1978. Hypothesis (EMH) is not rejected for lead and tin, while its is rejected for copper and zinc. Similarly, the examination of futures prices for copper and other metals indicated existence of systematic risk premiums or forecasting powers. The study on speculative efficiency of London Metal Exchange (LME) for six base metals during 1985-89 shows that the long-run speculative efficiency cannot be rejected for copper and

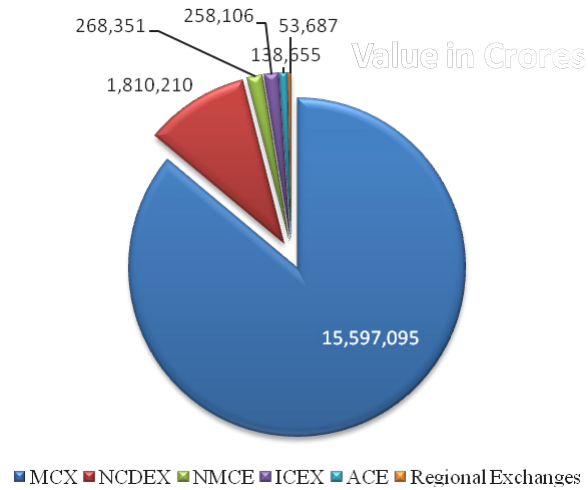

Figure 2. Commodity futures in india by exchange and traded value during 2011-12.

other three metals. On the other hand, the same hypothesis is rejected for the copper futures contract traded on the LME according to Chowdhury (1991) and Beck (1994). The study by Pantisa Pavabutr and Piyamas Chaihetphon (2010) shows that gold mini futures contracts at Multi Commodity Exchange of India (MCX) contribute to over $30 \%$ if price discovery in gold futures trade even though they account for only $2 \%$ of trading value on the MCX. Mini contracts are found to be more informative than the size of their market share of volume suggests. Garbade and Silber (1983) tested seven commodities, viz., Copper, Gold, Silver, Wheat, Corn, Oats and Frozen orange juices, for efficiency in risk management and price discovery. In their research, they argue that futures contracts will not in general, provide perfect risk transfer facilities over short-run horizons; though over the long run cash and futures prices should be integrated. But gold and silver on the contrary were highly integrated even over one day.

Studies by Naik and Jain (2002) and Lokare (2007) indicate progressive development of commodity market efficiency in metals with incidents of speculative activities in agricultural commodities. Empirical research by Dimitris F. Kenorgios (2004) once again upheld that copper futures markets on the London Metal Exchange are inefficient and the three and fifteen months of futures prices do not provide unbiased estimates of the future spot prices in both the long-run and short-run. Further [Turnovsky and Campbell (1985)], it was claimed that forward markets reduce the price risk of holding inventories, while, larger inventories are held and prices tend to stabilise as a consequence. Kawai (1983) showed that when storage is subject to shocks, increased storage can 
destabilise prices. It is also revealed that risk reduction encourages producers to undertake more risky investment projects, and risky investments destabilize spot prices. Similarly, Cox (1976) finds that in many markets, forward trading is stabilizing whereas Figlewski (1981) and Simpson and Ireland conclude that opposite is true. Varangis and Larson cited several examples in the case of cotton and oil in Mexico and Algeria, where group of producers is represented by an agent who trades on their behalf. In doing so, minimum prices for output could be guaranteed and thus, risk is reduced for an individual trader for the cost of a small premium.

\subsection{Objective of the Study}

The present study aims to study the operational efficiency of commodity derivatives in India in price risk management.

\subsection{Data and Methodology}

The present study is an analytical study based on secondary data consisting of daily spot and the nearest month future prices were taken for selected agricultural, metal and energy commodities traded in Indian derivatives market. Data for metals consisting of Aluminium, Copper, Gold, Silver, Nickel, and Zinc as well energy commodities - crude oil and natural gas is sourced from Multi Commodity Exchange India (MCX). Similarly 8 commodities consisting of Barley, Castor Seed, Guar Gum, Chana, Mustard Seed, Pepper, Potato and Soya bean is obtained from National Commodities and Derivatives Exchange of India (NCDEX) websites. The study covers a period of 5 years starting from $1^{\text {st }}$ Jan 2008 till 31 ${ }^{\text {st }}$ December 2012. Econometric tools like Augmented Dickey Fuller test and Co-integration Analysis is conducted to analyse the long-run relationship between the spot and futures prices of selected commodities. The study also uses ratio analysis of variability of spot, futures prices to study the existence of speculative and price risk in commodity derivative trading for selected commodities.

\section{Analysis and Discussion}

\subsection{Efficiency of Commodity Derivatives: Tests for Co-Integration}

Commodity derivative markets perform the important functions of price discovery and risk management.
Finance literature states that price insurance is possible only if spot and future prices move exactly together. In other words, if markets are efficient, there has to be co-movement between both the spot and future prices. In the finance literature, a pre-condition for market efficiency is the convergence of both future and spot prices across the market spectrum.

The proposition of risk reduction through hedging rests on the premise that the spot and future markets move together so that losses in one market can be made good through gains in other market. For the future price to be an unbiased predictor of the spot price, the future and spot prices must be proportional that is the basis should be constant and the market is said to be efficient. The studies exploring the price discovery role and the lead lag relationship between futures and spot prices have followed a procedure that is based on price series being non-stationary (Asche and Guttormsen, 2002), i.e., to test the existence of a long-run relationship between the spot and future prices by investigating whether the data series are co-integrated. In the present paper, Co-integration test developed by Johansen-Juselius is applied to test if the price data series are co-integrated.

Like most of the time series data, the price series of Indian commodity derivatives markets are nonstationary and exhibit the properties of random walk (no tendency to slip back to an underlying trend value). But differencing the data, makes the data stationary, but runs the risk of losing information about underlying long run relationships between the prices (Tables 6 to Table 8 in Annexures). Thus, the relationship and co-movement between the prices is examined in a cointegration framework in which linear combinations of non-stationary variables could be identified. Conducting the estimation of both future and spot prices under the Johansen-Juselius (JJ) procedure yields the following results (Table 3).

Co-integration analysis of spot and futures prices of selected 8 agricultural commodities indicates existence of co-integrating (long-term) relationship between the data series under consideration. Of all, castor seed and guar seed display two such co-integrating equations (relationships) thereby indicating higher operational efficiency and improved transmission of information 
in both spot and derivative prices. Conversely, price series of remaining six commodities namely barley, chana, mustard seed, pepper, potato and soya bean result in one co-integrating relationship.

Similarly, the results of JJ co-integration of spot and futures prices of metals show that Aluminium, Nickel and Copper have two co-integrating equations indicating higher operational efficiency than other metals which have one co-integrating equation. Even crude oil and natural gas price data series yield one cointegrating equation. So, it is evident from the results that the commodity derivative markets are inefficient in the long run (Table 4 and 5).

\subsection{Price Volatility}

For efficient markets, the extent of variations in spot and futures prices is expected to be same for storable com-

Table 3. Results of Johansen test for co-integration for agricultural spot and futures prices

\begin{tabular}{|c|c|c|c|c|c|c|}
\hline Commodity & Max-Eigen Value & $5 \%$ Critical Value & Trace Statistic & $5 \%$ Critical Value & Prob. Values & Hypothesised No. of Co integrating Equations \\
\hline \multirow[t]{2}{*}{ Barley } & 37.6953 & 14.26460 & 40.94531 & 15.4947 & 0.0000 & None* \\
\hline & 3.2499 & 3.841466 & 3.249996 & 3.8414 & 0.0714 & At Most 1 \\
\hline \multirow[t]{2}{*}{ Castor Seed } & 51.03392 & 14.26460 & 55.10140 & 15.4947 & 0.0000 & None $^{*}$ \\
\hline & 4.067480 & 3.841466 & 4.067480 & 3.8415 & 0.0437 & At Most $1^{*}$ \\
\hline \multirow[t]{2}{*}{ Chana } & 58.6132 & 14.26460 & 60.20884 & 15.4947 & 0.0000 & None $^{\star}$ \\
\hline & 1.5955 & 3.841466 & 1.595582 & 3.841466 & 0.2065 & At Most 1 \\
\hline \multirow[t]{2}{*}{ Guar Seed } & 79.9130 & 14.26460 & 86.5751 & 15.4947 & 0.0000 & None* $^{\star}$ \\
\hline & 6.6620 & 3.841466 & 6.662068 & 3.841466 & 0.0098 & At Most $1^{*}$ \\
\hline \multirow[t]{2}{*}{ Mustard Seed } & 43.9351 & 14.26460 & 98.35988 & 15.4947 & 0.0001 & None $^{*}$ \\
\hline & 0.2365 & 3.841466 & 0.661901 & 3.8415 & 0.4159 & At Most 1 \\
\hline \multirow[t]{2}{*}{ Pepper } & 43.9352 & 14.26460 & 44.17174 & 15.49471 & 0.0000 & None $^{*}$ \\
\hline & 0.2365 & 3.841466 & 0.236543 & 3.8415 & 0.6267 & At Most 1 \\
\hline \multirow[t]{2}{*}{ Potato } & 29.4593 & 14.26460 & 34.21601 & 15.49471 & 0.0000 & None $^{*}$ \\
\hline & 4.7566 & 3.841466 & 4.756689 & 3.841466 & 0.0292 & At Most $1^{*}$ \\
\hline \multirow[t]{2}{*}{ Soybean } & 60.5030 & 14.26460 & 63.06916 & 15.49471 & 0.0000 & None* \\
\hline & 2.5661 & 3.841466 & 2.566137 & 3.841466 & 0.1092 & At Most 1 \\
\hline
\end{tabular}

*: Denotes rejection of the hypothesis at the 0.05 level.

Table 4. Results of Johansen test for co-integration for metal spot and futures prices

\begin{tabular}{|c|c|c|c|c|c|c|}
\hline Commodity & Max-Eigen Value & $5 \%$ Critical Value & Trace Statistic & $5 \%$ Critical Value & Prob. Values & Hypothesised No. of Cointegrating Equations \\
\hline \multirow[t]{2}{*}{ Aluminium } & 28.88005 & 14.26460 & 33.18745 & 15.49471 & 0.0000 & None $^{*}$ \\
\hline & 4.307398 & 3.841466 & 4.307398 & 3.841466 & 0.0379 & At Most $1^{*}$ \\
\hline \multirow[t]{2}{*}{ Copper } & 125.8416 & 14.26460 & 128.0691 & 15.49471 & 0.0001 & None* $^{*}$ \\
\hline & 2.227522 & 3.841466 & 2.227522 & 3.841466 & 0.1356 & At Most $1^{*}$ \\
\hline \multirow[t]{2}{*}{ Gold } & 85.72242 & 14.26460 & 87.85180 & 15.49471 & 0.0000 & None* \\
\hline & 2.129378 & 3.841466 & 2.129378 & 3.841466 & 0.1445 & At Most 1 \\
\hline \multirow[t]{2}{*}{ Nickel } & 231.0857 & 14.26460 & 235.8632 & 15.49471 & 0.0001 & None* $^{*}$ \\
\hline & 4.777479 & 3.841466 & 4.777479 & 3.841466 & 0.0288 & At Most $1^{*}$ \\
\hline \multirow[t]{2}{*}{ Silver } & 81.42669 & 14.26460 & 83.43162 & 15.49471 & 0.0000 & None* $^{*}$ \\
\hline & 2.004931 & 3.841466 & 2.004931 & 3.841466 & 0.1568 & At Most 1 \\
\hline \multirow[t]{2}{*}{ Zinc } & 254.5968 & 14.26460 & 259.0464 & 15.49471 & 0.0001 & None* $^{\star}$ \\
\hline & 4.449619 & 3.841466 & 4.449619 & 3.841466 & 0.0349 & At Most 1 \\
\hline
\end{tabular}

*: Denotes rejection of the hypothesis at the 0.05 level. 
Table 5. Results of Johansen test for co-integration for energy spot and futures prices

\begin{tabular}{lcccccc}
\hline Commodity & Max-Eigen Value & $5 \%$ Critical Value & Trace Statistic & $5 \%$ Critical Value & Prob. Values & Hypothesised No. of Cointegrating Equations \\
\hline Crude Oil & 255.0626 & 14.26460 & 256.5957 & 15.49471 & 0.0001 & None* $^{*}$ \\
& 1.533083 & 3.841466 & 1.533083 & 3.841466 & 0.2156 & At Most $1^{*}$ Natural Gas \\
& 326.4139 & 14.26460 & 329.0476 & 15.49471 & 0.0001 & None* $^{*}$ \\
& 2.633793 & 3.841466 & 2.633793 & 3.841466 & 0.1046 & At Most 1 \\
\hline
\end{tabular}

*: Denotes rejection of the hypothesis at the 0.05 level.

Table 6. Results of ADF test for stationarity in prices of agricultural commodities in NCDEX

\begin{tabular}{lllc}
\hline Commodity & Price & At Level & 1st Differencing \\
\hline Barley & Future & 0.1236 & $0.0000^{*}$ \\
& Spot & 0.2530 & $0.0000^{*}$ \\
Castor Seed & Future & 0.2256 & $0.0000^{*}$ \\
& Spot & 0.2184 & $0.0000^{*}$ \\
Chana & Future & 0.5827 & $0.0000^{*}$ \\
& Spot & 0.6820 & $0.0000^{*}$ \\
Guar Seed & Future & 0.0966 & $0.0000^{*}$ \\
& Spot & 0.0704 & $0.0000^{*}$ \\
Mustard Seed & Future & 0.8002 & $0.0000^{*}$ \\
& Spot & 0.8330 & $0.0000^{*}$ \\
Pepper & Future & 0.8285 & $0.0000^{*}$ \\
& Spot & 0.8872 & $0.0000^{*}$ \\
Potato & Future & 0.2705 & $0.0000^{*}$ \\
& Spot & 0.3173 & $0.0000^{*}$ \\
Soybean & Future & 0.6362 & $0.0000^{*}$ \\
& Spot & 0.6620 & $0.0000^{*}$ \\
\hline *Significant at $5 \%$ level & &
\end{tabular}

modities. In other words, if the spot market is efficient, understanding of relative variations in prices help us to see whether futures market is able to assimilate the information efficiently. Moreover the daily variations in spot and derivatives come purely from new information that enters the market.

The ratio of standard deviations of month-wise daily future and spot prices throws light on the extent of volatility in the derivative markets. Assuming that the carrying costs in the month are negligible, a ratio of standard deviation of future and spot prices that is closer to one indicates that derivatives market is efficient, viz., markets are incorporating the information efficiently. A ratio greater than one close to the maturity period indicates speculative activities. Conversely, a ratio less than one shows that markets
Table 7. Results of ADF test for stationarity in prices of energy commodities in MCX

\begin{tabular}{lllc}
\hline Commodity & Price & At Level & 1st Differencing \\
\hline Crude 0il & Future & 0.6783 & $0.0000^{*}$ \\
& Spot & 0.5912 & $0.0000^{\star}$ \\
Natural Gas & Future & 0.3360 & $0.0000^{*}$ \\
& Spot & 0.4151 & $0.0001^{*}$ \\
\hline *Signifant $5 \%$ & &
\end{tabular}

Table 8. Results of ADF test for stationarity in prices of metal commodities in MCX

\begin{tabular}{lllc}
\hline Commodity & Price & At Level & 1st Differencing \\
\hline Aluminium & Future & 0.3117 & $0.0000^{*}$ \\
& Spot & 0.4940 & $0.0000^{*}$ \\
Copper & Future & 0.5643 & $0.0000^{*}$ \\
& Spot & 0.5561 & $0.0000^{*}$ \\
Gold & Future & 0.5601 & $0.0000^{*}$ \\
& Spot & 0.5690 & $0.0000^{*}$ \\
Nickel & Future & 0.2275 & $0.0000^{\star}$ \\
& Spot & 0.2084 & $0.0000^{*}$ \\
Silver & Future & 0.5935 & $0.0000^{*}$ \\
& Spot & 0.6020 & $0.0000^{*}$ \\
Zinc & Future & 0.2575 & $0.0000^{*}$ \\
& Spot & 0.2470 & $0.0000^{*}$ \\
\hline *Significant at $5 \%$ level & &
\end{tabular}

are not being able to incorporate the information fully and efficiently. 0.8 and 1.2 has been assumed as the lower and upper levels to provide an indication of extent of variability in the spot and derivatives markets (K.M. Lokare (2007), Naik and Jain (2002)). The results of analysis are presented below. (Tables 9-24 for results of analysis in Annexures)

- For Barley, in large number of cases the ratio has exceeded an upper limit of 1.2 and in few cases reached the peak levels of 6-8 times which indicates 
Table 9. Ratio Between Standard Deviation of Future and Spot Prices of Barley

\begin{tabular}{cccccccccccccccc}
\hline & & & & & & & & & & & & & \multicolumn{3}{c}{ No of Times } \\
\cline { 9 - 15 } & Jean & Feb & Mar & Apr & May & Jun & Jul & Aug & Sep & Oct & Nov & Dec & $<0.8$ & $>1.2$ & $0.8-1.2$ \\
\hline 2008 & 2.89 & 1.27 & 0.93 & 0.73 & 1.29 & 1.40 & 1.19 & 1.37 & 2.27 & 1.15 & 1.41 & 0.70 & 2 & 7 & 3 \\
2009 & 0.97 & 1.07 & 0.59 & 1.04 & 0.96 & 1.30 & 1.14 & 1.63 & 1.39 & 1.66 & 1.29 & 8.02 & 1 & 6 & 5 \\
2010 & 2.52 & 3.96 & 2.08 & 0.98 & 1.49 & 1.60 & 1.11 & 1.27 & 1.08 & 1.25 & 2.97 & 6.32 & 0 & 9 & 3 \\
2011 & 3.36 & 3.45 & 0.68 & 1.18 & 0.65 & 0.97 & 2.82 & 2.06 & 2.55 & 2.04 & 2.14 & 3.21 & 2 & 8 & 2 \\
2012 & 1.41 & 6.68 & 0.71 & 1.16 & 1.43 & 0.89 & 1.37 & 1.25 & 2.00 & 2.32 & 1.22 & 2.55 & 1 & 9 & 2 \\
\hline
\end{tabular}

Table 10. Ratio Between Standard Deviation of Future and Spot Prices of Castor Seed

\begin{tabular}{cccccccccccccccc}
\hline Year & Jan & Feb & Mar & Apr & May & Jun & Jul & Aug & Sep & Oct & Nov & Dec & $<0.8$ & $>1.2$ & $0.8-1.2$ \\
\hline 2008 & - & - & - & - & - & - & - & - & - & - & - & - & 0 & 0 & 0 \\
2009 & - & - & - & - & - & - & - & - & - & 1.83 & 0.99 & 1.04 & & 1 & 11 \\
2010 & 1.38 & 0.91 & 1.54 & 1.05 & 0.66 & 1.26 & 0.85 & 1.11 & 0.79 & 1.03 & 1.00 & 0.87 & 2 & 3 & 7 \\
2011 & 0.74 & 0.87 & 0.83 & 0.73 & 1.18 & 1.50 & 1.08 & 1.37 & 1.06 & 0.85 & 1.42 & 1.55 & 2 & 4 & 6 \\
2012 & 1.10 & 0.92 & 1.21 & 0.97 & 1.07 & 1.15 & 1.17 & 1.56 & 1.06 & 1.95 & 1.50 & 1.08 & 0 & 4 & 8 \\
\hline
\end{tabular}

Table 11. Ratio Between Standard Deviation of Future and Spot Prices of Chana

\begin{tabular}{|c|c|c|c|c|c|c|c|c|c|c|c|c|c|c|c|}
\hline \multirow[b]{2}{*}{ Year } & \multirow[b]{2}{*}{ Jan } & \multirow[b]{2}{*}{ Feb } & \multirow[b]{2}{*}{ Mar } & \multirow[b]{2}{*}{ Apr } & \multirow[b]{2}{*}{ May } & \multirow[b]{2}{*}{ Jun } & \multirow[b]{2}{*}{ Jul } & \multirow[b]{2}{*}{ Aug } & \multirow[b]{2}{*}{ Sep } & \multirow[b]{2}{*}{ Oct } & \multirow[b]{2}{*}{ Nov } & \multirow[b]{2}{*}{ Dec } & \multicolumn{3}{|c|}{ No of Times } \\
\hline & & & & & & & & & & & & & $<0.8$ & $>1.2$ & $0.8-1.2$ \\
\hline 2008 & 2.89 & 0.87 & 1.20 & 0.78 & 0.67 & - & - & - & - & - & - & 0.77 & 9.00 & 2 & 1 \\
\hline 2009 & 1.38 & 1.26 & 0.79 & 0.99 & 1.55 & 1.11 & 1.10 & 1.82 & 2.07 & 1.46 & 1.15 & 2.52 & 1.00 & 7 & 4 \\
\hline 2010 & 1.36 & 0.89 & 1.57 & 1.12 & 1.63 & 1.18 & 1.41 & 2.27 & 1.88 & 1.64 & 1.25 & 1.14 & - & 8 & 4 \\
\hline 2011 & 0.66 & 1.43 & 0.46 & 1.73 & 1.00 & 1.15 & 1.24 & 1.06 & 0.90 & 1.45 & 1.07 & 1.13 & 2.00 & 4 & 6 \\
\hline 2012 & 1.44 & 0.94 & 0.79 & 0.97 & 0.79 & 1.39 & 0.78 & 1.23 & 0.91 & 1.14 & 2.26 & 0.99 & 3.00 & 4 & 5 \\
\hline
\end{tabular}

Table 12. Ratio Between Standard Deviation of Future and Spot Prices of Guar Seed

\begin{tabular}{lccccccccccccccc}
\hline & & & & & & & & & & & & & & \multicolumn{4}{c}{ No of Times } \\
\cline { 10 - 17 } Year & Jan & Feb & Mar & Apr & May & Jun & Jul & Aug & Sep & Oct & Nov & Dec & $<0.8$ & $>1.2$ & $0.8-1.2$ \\
\hline 2008 & 1.76 & 0.97 & 1.96 & 0.75 & 1.56 & 1.66 & 1.63 & 1.43 & 1.16 & 1.14 & 1.41 & 1.41 & 1 & 8 & 3 \\
2009 & 2.07 & 1.42 & 1.85 & 1.17 & 1.54 & 1.85 & 1.20 & 1.31 & 1.41 & 0.92 & 1.56 & 1.36 & 0 & 10 & 2 \\
2010 & 1.19 & 1.19 & 1.07 & 1.48 & 1.20 & 1.14 & 1.27 & 1.15 & 1.17 & 1.38 & 1.20 & 0.88 & 0 & 4 & 8 \\
2011 & 1.09 & 1.26 & 1.31 & 1.57 & 0.76 & 1.04 & 1.00 & 1.03 & 1.13 & 1.37 & 1.28 & 1.06 & 1 & 5 & 6 \\
2012 & 0.91 & 0.99 & 0.94 & - & 1.34 & 0.99 & - & - & - & - & - & - & - & 1 & 4 \\
\hline
\end{tabular}

Table 13. Ratio Between Standard Deviation of Future and Spot Prices of Mustard Seed

\begin{tabular}{|c|c|c|c|c|c|c|c|c|c|c|c|c|c|c|c|}
\hline \multirow[b]{2}{*}{ Year } & \multirow[b]{2}{*}{ Jan } & \multirow[b]{2}{*}{ Feb } & \multirow[b]{2}{*}{ Mar } & \multirow[b]{2}{*}{ Apr } & \multirow[b]{2}{*}{ May } & \multirow[b]{2}{*}{ Jun } & \multirow[b]{2}{*}{ Jul } & \multirow[b]{2}{*}{ Aug } & \multirow[b]{2}{*}{ Sep } & \multirow[b]{2}{*}{ Oct } & \multirow[b]{2}{*}{ Nov } & \multirow[b]{2}{*}{ Dec } & \multicolumn{3}{|c|}{ No of Times } \\
\hline & & & & & & & & & & & & & $<0.8$ & $>1.2$ & $0.8-1.2$ \\
\hline 2008 & 1.19 & 2.34 & 1.09 & 1.14 & 1.56 & 0.52 & 1.41 & 1.56 & 1.25 & 1.45 & 2.42 & 0.66 & 2 & 7 & 3 \\
\hline 2009 & 1.54 & 1.54 & 0.71 & 1.11 & 0.91 & 1.26 & 1.06 & 0.96 & 1.52 & 1.20 & 0.80 & 1.22 & 2 & 5 & 5 \\
\hline 2010 & 1.56 & 0.58 & 1.37 & 0.94 & 1.08 & 0.90 & 1.21 & 1.20 & 1.42 & 1.59 & 0.76 & 1.77 & 2 & 6 & 4 \\
\hline 2011 & 1.22 & 5.78 & 0.68 & 1.12 & 0.78 & 1.45 & 0.99 & 1.43 & 1.82 & 1.97 & 0.90 & 1.32 & 2 & 7 & 3 \\
\hline 2012 & 1.38 & 1.05 & 0.84 & 1.36 & 1.83 & 1.84 & 0.92 & 0.96 & 1.29 & 1.20 & 0.95 & 1.10 & 0 & 5 & 7 \\
\hline
\end{tabular}


Table 14. Ratio Between Standard Deviation of Future and Spot Prices of Pepper

\begin{tabular}{|c|c|c|c|c|c|c|c|c|c|c|c|c|c|c|c|}
\hline \multirow[b]{2}{*}{ Year } & \multirow[b]{2}{*}{ Jan } & \multirow[b]{2}{*}{ Feb } & \multirow[b]{2}{*}{ Mar } & \multirow[b]{2}{*}{ Apr } & \multirow[b]{2}{*}{ May } & \multirow[b]{2}{*}{ Jun } & \multirow[b]{2}{*}{ Jul } & \multirow[b]{2}{*}{ Aug } & \multirow[b]{2}{*}{ Sep } & \multirow[b]{2}{*}{ Oct } & \multirow[b]{2}{*}{ Nov } & \multirow[b]{2}{*}{ Dec } & \multicolumn{3}{|c|}{ No of Times } \\
\hline & & & & & & & & & & & & & $<0.8$ & $>1.2$ & $0.8-1.2$ \\
\hline 2008 & 1.09 & 1.59 & 1.89 & 1.45 & 1.52 & 1.52 & 1.97 & 2.22 & 1.32 & 0.99 & 1.38 & 0.92 & 0 & 9 & 3 \\
\hline 2009 & 1.02 & 1.07 & 1.29 & 0.98 & 1.37 & 1.33 & 1.71 & 1.01 & 1.47 & 1.50 & 1.82 & 1.33 & 0 & 8 & 4 \\
\hline 2010 & 1.34 & 1.36 & 1.35 & 1.54 & 1.43 & 1.20 & 1.01 & 1.44 & 1.40 & 1.39 & 1.31 & 1.58 & 0 & 11 & 1 \\
\hline 2011 & 1.96 & 1.53 & 1.63 & 1.05 & 1.39 & 1.86 & 1.41 & 1.47 & 1.04 & 1.62 & 2.51 & 1.69 & 0 & 10 & 2 \\
\hline 2012 & 1.41 & 1.49 & 1.27 & 1.56 & 1.62 & 1.28 & 1.34 & 1.56 & 1.67 & 1.84 & 1.34 & 5.68 & 0 & 12 & 0 \\
\hline
\end{tabular}

Table 15. Ratio Between Standard Deviation of Future and Spot Prices of Potato

\begin{tabular}{|c|c|c|c|c|c|c|c|c|c|c|c|c|c|c|c|}
\hline \multirow[b]{2}{*}{ Year } & \multirow[b]{2}{*}{ Jan } & \multirow[b]{2}{*}{ Feb } & \multirow[b]{2}{*}{ Mar } & \multirow[b]{2}{*}{ Apr } & \multirow[b]{2}{*}{ May } & \multirow[b]{2}{*}{ Jun } & \multirow[b]{2}{*}{ Jul } & \multirow[b]{2}{*}{ Aug } & \multirow[b]{2}{*}{ Sep } & \multirow[b]{2}{*}{ Oct } & \multirow[b]{2}{*}{ Nov } & \multirow[b]{2}{*}{ Dec } & \multicolumn{3}{|c|}{ No of Times } \\
\hline & & & & & & & & & & & & & $<0.8$ & $>1.2$ & $0.8-1.2$ \\
\hline 2008 & - & - & 0.85 & 0.66 & 1.21 & - & - & - & - & - & - & - & 1 & 1 & 1 \\
\hline 2009 & - & -0.51 & 1.10 & 1.33 & 1.18 & 4.12 & 1.68 & 0.68 & - & - & - & & 2 & 3 & 2 \\
\hline 2010 & 1.00 & 0.97 & 0.86 & 0.48 & 0.85 & 3.08 & 1.10 & 1.41 & 1.75 & 0.80 & 0.72 & 1.00 & 3 & 3 & 6 \\
\hline 2011 & 1.00 & 1.00 & 1.70 & 2.22 & 2.88 & 1.93 & 2.80 & 1.14 & 7.87 & 0.90 & 0.11 & 1.00 & 1 & 6 & 5 \\
\hline 2012 & 1.00 & 1.00 & 1.48 & 0.54 & 4.64 & 6.36 & 1.10 & 2.25 & 0.57 & 1.76 & 0.09 & 0.75 & 4 & 5 & 3 \\
\hline
\end{tabular}

Table 16. Ratio Between Standard Deviation of Future and Spot Prices of Soyabean

\begin{tabular}{|c|c|c|c|c|c|c|c|c|c|c|c|c|c|c|c|}
\hline \multirow[b]{2}{*}{ Year } & \multirow[b]{2}{*}{ Jan } & \multirow[b]{2}{*}{ Feb } & \multirow[b]{2}{*}{ Mar } & \multirow[b]{2}{*}{ Apr } & \multirow[b]{2}{*}{ May } & \multirow[b]{2}{*}{ Jun } & \multirow[b]{2}{*}{ Jul } & \multirow[b]{2}{*}{ Aug } & \multirow[b]{2}{*}{ Sep } & \multirow[b]{2}{*}{ Oct } & \multirow[b]{2}{*}{ Nov } & \multirow[b]{2}{*}{ Dec } & \multicolumn{3}{|c|}{ No of Times } \\
\hline & & & & & & & & & & & & & $<0.8$ & $>1.2$ & $0.8-1.2$ \\
\hline 2008 & 0.98 & 1.12 & 1.23 & 1.14 & 1.09 & 1.40 & 2.49 & 1.04 & 0.79 & 0.87 & 1.00 & 0.95 & 1 & 3 & 8 \\
\hline 2009 & 0.93 & 1.48 & 1.09 & 0.81 & 1.80 & 1.04 & 1.02 & 1.08 & 1.29 & 1.04 & 0.89 & 1.08 & 0 & 3 & 9 \\
\hline 2010 & 1.19 & 1.08 & 1.37 & 0.77 & 0.96 & 1.16 & 1.20 & 1.50 & 1.09 & 0.93 & 1.41 & 1.19 & 1 & 4 & 7 \\
\hline 2011 & 1.14 & 0.96 & 1.28 & 1.03 & 1.43 & 1.67 & 1.50 & 1.15 & 1.05 & 1.46 & 0.96 & 2.17 & 0 & 6 & 6 \\
\hline 2012 & 1.39 & 1.22 & 1.11 & 1.42 & 1.28 & 0.96 & 5.48 & 0.55 & 0.97 & 1.08 & 1.14 & 1.07 & 1 & 5 & 6 \\
\hline
\end{tabular}

Table 17. Ratio Between Standard Deviation of Future and Spot Prices of Aluminium

\begin{tabular}{lccccccccccccccc}
\hline Year & Jan & Feb & Mar & Apr & May & Jun & Jul & Aug & Sep & Oct & Nov & Dec & $<0.8$ & $>1.2$ & $0.8-1.2$ \\
\hline 2008 & 2.33 & 1.64 & 1.12 & 0.69 & 1.94 & 1.46 & 1.77 & 0.27 & 0.91 & 3.36 & 1.11 & 1.04 & 2 & 6 & 4 \\
2009 & 0.91 & 1.00 & 0.44 & 1.07 & 2.18 & 2.49 & 2.49 & 0.72 & 1.22 & 0.46 & 0.64 & 1.83 & 4 & 5 & 3 \\
2010 & 1.25 & 0.68 & 0.48 & 1.38 & 1.29 & 0.77 & 0.96 & 0.97 & 0.43 & 0.57 & 2.06 & 1.43 & 5 & 5 & 2 \\
2011 & 0.79 & 0.43 & 0.64 & 1.13 & 1.23 & 1.11 & 1.19 & 2.03 & 1.02 & 1.37 & 0.74 & 0.66 & 5 & 3 & 4 \\
2012 & 0.65 & 0.98 & 0.73 & 0.52 & 0.79 & 0.88 & 0.50 & 0.74 & 1.74 & 1.10 & 1.69 & 0.61 & 7 & 2 & 3 \\
\hline
\end{tabular}

Table 18. Ratio Between Standard Deviation of Future and Spot Prices of Copper

\begin{tabular}{lccccccccccccccc}
\hline Year & Jan & Feb & Mar & Apr & May & Jun & Jul & Aug & Sep & Oct & Nov & Dec & $<0.8$ & $>1.2$ & $0.8-1.2$ \\
\hline 2008 & 0.96 & 0.97 & 0.89 & 0.91 & 0.85 & 1.02 & 0.84 & 0.88 & 0.68 & 0.86 & 0.96 & 0.99 & 1 & - & 11 \\
2009 & 0.76 & 0.92 & 0.87 & 1.00 & 0.91 & 0.88 & 0.96 & 0.91 & 0.95 & 0.96 & 0.97 & 0.99 & 1 & - & 11 \\
2010 & 1.11 & 0.91 & 0.84 & 1.02 & 0.82 & 0.84 & 0.91 & 0.97 & 0.83 & 1.04 & 0.92 & 1.02 & - & - & 12 \\
2011 & 1.10 & 1.02 & 0.95 & 1.23 & 0.88 & 1.06 & 0.86 & 0.91 & 1.02 & 0.93 & 1.15 & 0.90 & - & 1 & 11 \\
2012 & 0.88 & 0.88 & 0.76 & 0.94 & 0.94 & 1.10 & 0.57 & 0.86 & 0.80 & 0.90 & 0.85 & 0.91 & 3 & - & 9 \\
\hline
\end{tabular}


Table 19. Ratio Between Standard Deviation of Future and Spot Prices of Gold

\begin{tabular}{cccccccccccccccc}
\hline Year & Jan & Feb & Mar & Apr & May & Jun & Jul & Aug & Sep & Oct & Nov & Dec & $<0.8$ & $>1.2$ & $0.8-1.2$ \\
\hline 2008 & 1.04 & 1.04 & 1.03 & 1.03 & 1.01 & 1.00 & 1.00 & 1.00 & 1.00 & 0.99 & 1.00 & 0.99 & - & - & 12 \\
2009 & 1.00 & 1.01 & 1.02 & 1.02 & 1.02 & 1.01 & 1.02 & 1.02 & 1.02 & 1.02 & 1.02 & 1.01 & - & - & 12 \\
2010 & 1.02 & 1.01 & 1.01 & 1.02 & 1.02 & 1.02 & 1.02 & 1.01 & 1.01 & 1.00 & 1.00 & 1.00 & - & - & 12 \\
2011 & 1.00 & 1.00 & 0.99 & 1.00 & 1.00 & 1.01 & 1.01 & 1.01 & 1.01 & 1.01 & 1.01 & 1.01 & - & - & 12 \\
2012 & 1.01 & 1.01 & 1.01 & 1.01 & 1.01 & 1.01 & 1.00 & 1.00 & 1.00 & 1.00 & 1.01 & 1.01 & - & - & 12 \\
\hline
\end{tabular}

Table 20. Ratio Between Standard Deviation of Future and Spot Prices of Guar Silver

\begin{tabular}{cccccccccccccccc}
\hline Year & Jan & Feb & Mar & Apr & May & Jun & Jul & Aug & Sep & Oct & Nov & Dec & $<0.8$ & $>1.2$ & $0.8-1.2$ \\
\hline 2008 & 1.25 & 1.23 & 1.73 & 1.06 & 1.09 & 0.95 & 1.30 & 0.98 & 1.06 & 0.98 & 0.94 & 1.22 & - & 5 & 7 \\
2009 & 1.13 & 0.99 & 1.57 & 1.11 & 1.32 & 1.61 & 1.04 & 1.06 & 1.10 & 1.13 & 0.92 & 1.03 & - & 3 & 9 \\
2010 & 1.13 & 0.99 & 0.82 & 0.97 & 0.94 & 0.87 & 0.93 & 1.27 & 0.89 & 0.99 & 1.05 & 1.04 & - & 1 & 11 \\
2011 & 0.84 & 1.02 & 1.00 & 0.93 & 0.95 & 1.07 & 1.08 & 0.95 & 1.14 & 1.31 & 1.17 & 0.91 & - & 1 & 11 \\
2012 & 1.06 & 0.89 & 0.80 & 1.77 & 0.87 & 1.15 & 0.86 & 1.00 & 1.60 & 1.44 & 0.99 & 1.06 & - & 3 & 9 \\
\hline
\end{tabular}

Table 21. Ratio Between Standard Deviation of Future and Spot Prices of Nickel

\begin{tabular}{cccccccccccccccc}
\hline Year & Jan & Feb & Mar & Apr & May & Jun & Jul & Aug & Sep & Oct & Nov & Dec & $<0.8$ & $>1.2$ & $0.8-1.2$ \\
\hline 2008 & 0.88 & 0.96 & 1.04 & 0.81 & 1.05 & 1.03 & 1.03 & 0.91 & 1.05 & 0.90 & 1.28 & 1.08 & - & 1 & 11 \\
2009 & 0.90 & 1.03 & 1.05 & 0.90 & 0.93 & 0.87 & 0.94 & 0.72 & 0.94 & 0.77 & 0.99 & 1.03 & 2 & - & 10 \\
2010 & 1.05 & 1.01 & 0.95 & 0.92 & 0.98 & 0.96 & 0.98 & 0.90 & 0.83 & 1.04 & 0.94 & 1.00 & - & - & 12 \\
2011 & 0.95 & 0.79 & 0.95 & 1.14 & 1.11 & 1.03 & 0.90 & 1.06 & 1.04 & 1.02 & 1.19 & 0.97 & 1 & - & 11 \\
2012 & 0.85 & 1.07 & 1.15 & 0.78 & 0.82 & 1.12 & 0.93 & 0.79 & 0.89 & 0.99 & 0.83 & 0.92 & 2 & - & 10 \\
\hline
\end{tabular}

Table 22. Ratio Between Standard Deviation of Future and Spot Prices of Zinc

\begin{tabular}{cccccccccccccccc}
\hline Year & Jan & Feb & Mar & Apr & May & Jun & Jul & Aug & Sep & Oct & Nov & Dec & $<0.8$ & $>1.2$ & $0.8-1.2$ \\
\hline 2008 & 0.87 & 1.06 & 0.98 & 1.29 & 0.97 & 0.98 & 0.95 & 0.99 & 1.02 & 0.96 & 0.76 & 0.86 & 1 & 1 & 10 \\
2009 & 1.08 & 0.97 & 0.94 & 0.99 & 0.98 & 0.93 & 0.87 & 0.97 & 0.95 & 0.93 & 0.88 & 1.00 & - & - & 12 \\
2010 & 1.02 & 1.12 & 1.08 & 0.93 & 1.03 & 1.05 & 0.92 & 0.87 & 1.03 & 0.96 & 0.99 & 0.87 & - & - & 12 \\
2011 & 1.06 & 0.79 & 1.01 & 1.06 & 0.94 & 1.12 & 0.89 & 1.12 & 1.08 & 1.07 & 0.82 & 0.99 & 1 & - & 11 \\
2012 & 0.85 & 0.91 & 0.92 & 0.70 & 0.94 & 0.82 & 0.69 & 0.94 & 0.83 & 1.06 & 0.84 & 0.82 & 2 & - & 10 \\
\hline
\end{tabular}

Table 23. Ratio Between Standard Deviation of Future and Spot Prices of Crude Oil

\begin{tabular}{cccccccccccccccc}
\hline Year & Jan & Feb & Mar & Apr & May & Jun & Jul & Aug & Sep & Oct & Nov & Dec & $<0.8$ & $>1.2$ & $0.8-1.2$ \\
\hline 2008 & 1.03 & 0.97 & 1.08 & 0.88 & 0.99 & 0.96 & 0.94 & 0.92 & 0.92 & 0.91 & 0.92 & 0.94 & - & - & 12 \\
2009 & 1.05 & 0.96 & 1.08 & 0.91 & 0.96 & 1.05 & 1.02 & 0.91 & 0.93 & 1.00 & 1.03 & 1.00 & - & - & 12 \\
2010 & 1.00 & 1.01 & 0.90 & 1.06 & 1.04 & 0.86 & 1.00 & 0.97 & 0.95 & 0.95 & 0.88 & 1.09 & - & - & 12 \\
2011 & 1.01 & 1.13 & 0.96 & 1.09 & 0.86 & 0.92 & 1.07 & 0.90 & 1.02 & 0.95 & 1.01 & 1.17 & - & - & 12 \\
2012 & 0.98 & 1.04 & 0.90 & 0.97 & 0.97 & 0.63 & 1.04 & 0.87 & 0.97 & 0.91 & 0.93 & 0.99 & 1 & - & 11 \\
\hline
\end{tabular}


Table 24. Ratio Between Standard Deviation of Future and Spot Prices of Natural Gas

\begin{tabular}{cccccccccccccccc}
\hline Year & Jan & Feb & Mar & Apr & May & Jun & Jul & Aug & Sep & Oct & Nov & Dec & $<0.8$ & $>1.2$ & $0.8-1.2$ \\
\hline 2008 & 0.95 & 1.02 & 0.97 & 0.98 & 0.94 & 0.86 & 1.00 & 0.91 & 1.03 & 1.08 & 0.96 & 0.94 & - & - & 12 \\
2009 & 1.03 & 1.06 & 0.90 & 0.99 & 0.96 & 0.89 & 0.99 & 1.06 & 1.08 & 1.03 & 0.97 & 0.96 & - & - & 12 \\
2010 & 0.96 & 1.11 & 0.95 & 0.90 & 0.98 & 0.85 & 1.06 & 0.89 & 1.12 & 1.17 & 1.00 & 1.08 & - & - & 12 \\
2011 & 0.92 & 0.98 & 1.01 & 1.07 & 1.02 & 1.02 & 0.96 & 1.01 & 0.95 & 1.01 & 0.90 & 0.99 & - & - & 12 \\
2012 & 1.02 & 0.97 & 0.99 & 1.14 & 0.94 & 0.98 & 1.04 & 0.87 & 1.02 & 1.11 & 0.95 & 1.06 & - & - & 12 \\
\hline
\end{tabular}

higher volatility in future price than in the spot prices. It may be assumed that speculative activities are very high in the case of barley especially between the months December to March.

- In the case of castor seed, in more than $60 \%$ of the cases the ration was closer to one, indicating that there is an efficient utilization of information to an extent and market is efficient for castor seed. However, contracts between October to January appear to attract high speculative trading.

- Chana also in around $45 \%$ cases attracts speculative activities with around $25 \%$ of the cases exhibiting efficient utilisation of information. It also appears to attract speculative activities between months October to January.

- Similarly, in case of guar seed and mustard seeds, around $40 \%$ of the cases of the ratio were closer to one. However, in large number of cases it was over the upper limit indicating existence of moderate levels of speculations.

- In the case of pepper, ratio has hovered around one in many cases, indicating that there is an efficient utilisation of information and to an extent market is efficient in respect of pepper. Among all the months, however, contracts between June to October to December appear to attract some speculative trading.

- Ratio of standard deviations for potato indicate that markets are efficient around $40 \%$ of the cases and of the remainder in large number of cases prices indicate existence of speculative activities moderately higher during May to September.

- In the case of Soya bean, around $60 \%$ of the ratio exhibited efficient utilisation of information and in remaining times ratio indicates existence of some speculative activities.

- In case of aluminium, the ratio indicates existence of speculative activities during the year 2008 but in later periods the ratio indicates the under utilisation of information in large number of cases.

- In case of Copper, Gold, Nickel and Zinc, the ratio was within the permissible limits indicating the highest level of information utilisation and efficiency. Among these metals, informational efficiency was very high in the case of Gold.

- Similarly, the ratio of deviations of futures and spot prices for silver exhibits higher levels of informational efficiency except in few months of 2008 and 2009 (where some speculative activities took place).

- In cases of crude oil and natural gas, the ratios indicated almost perfect information utilisation of market information and co-movement between the futures and spot prices.

\subsection{Basis Risk-Marginal Convenience Yield}

Basis is defined as the difference between the spot and futures contract price. If the spot price is more than the future price, the market is said to be in backwardation. On the other hand if the future price is greater than the spot price it is said to be in contango. On the expiry of future contracts, the spot and future prices are expected to become equal. The difference between the future price and spot price gives the direct measure of the marginal value of the storage for a commodity termed as Marginal Convenience Yield (MCY). If MCY is high, the spot prices are expected to exceed future prices. The level of inventories held in the spot market will be determined by the basis and will ensure a more efficient process of private storage, which in turn, ensures a smoother pattern of prices in the spot market and hence, potentially reduce price volatility (Netz, 1995 and Morgan, 1999). So the producers in commodity derivative markets face the basis risk. Lower basis risk indicates higher efficiency in price risk management by derivative markets. In other 
words, if the futures price converge with spot price in month of maturity resulting in zero basis, the markets are considered as efficient. In such markets, producer who hedges his price risk can contain his business risk by holding on to the contract until the maturity of the contract. Thus, if the basis is low, hedging becomes an effective instrument of price risk management. The effectiveness of commodity derivatives markets in terms of the price risk management could be examined by analysing the ratio of standard deviation of basis to the spot price in the maturity month of the contract. A ratio of standard deviation of basis to the spot price of any contract that is less than 0.5 (a benchmark) could be considered to be effective in price risk management and hence, would attract more participants to the derivatives market (Naik and Jain, 2002 and L.M. Lokare, 2007). The findings of analysis are presented below. (Tables 25 - 40 - results of analysis in Annexures)

- In the case of Barley, the ratio between Standard deviation of basis and spot prices indicate that very high basis risk as more than $85 \%$ of the times the ratio was above bench mark range of 0.5 . It is also evident that the basis risk is on raise after 2009.

- In case of castor seed and chana, for about $40 \%$ of the cases the ratio was below benchmark so it indicates moderate level of risk for the hedgers.

- Similarly, in guar seed and mustard seed, the ratio was matching the bench mark in around $45 \%$ and

Table 25. Ratio Between Standard Deviation of Basis and Spot Prices of Barley

\begin{tabular}{cccccccccccccccccc}
\hline Year & Jan & Feb & Mar & Apr & May & Jun & Jul & Aug & Sep & Oct & Nov & Dec & $>1$ & $0.5-1.0$. & $<0.5$ & $<1$ \\
\hline 2008 & 5.19 & 4.20 & 1.91 & 0.42 & 0.34 & 0.82 & 0.81 & 0.73 & 2.24 & 0.29 & 0.64 & 0.44 & 4 & 4 & 4 & 8 \\
2009 & 0.15 & 0.21 & 0.79 & 0.50 & 1.38 & 0.86 & 1.43 & 1.45 & 1.47 & 0.85 & 1.32 & 7.67 & 6 & 3 & 3 & 6 \\
2010 & 2.23 & 3.50 & 2.29 & 0.47 & 0.72 & 1.00 & 0.68 & 1.01 & 0.61 & 1.58 & 2.78 & 5.88 & 7 & 4 & 1 & 5 \\
2011 & 2.72 & 3.82 & 0.52 & 0.47 & 0.65 & 0.83 & 2.22 & 1.19 & 2.31 & 2.60 & 2.11 & 2.51 & 8 & 3 & 1 & 4 \\
2012 & 1.20 & 6.00 & 0.44 & 0.66 & 0.51 & 1.12 & 0.58 & 0.69 & 1.56 & 1.41 & 0.51 & 1.84 & 6 & 5 & 1 & 6 \\
\hline
\end{tabular}

Table 26. Ratio Between Standard Deviation of Basis and Spot Prices of Castor Seed

\begin{tabular}{ccccccccccccccccc}
\hline Year & Jan & Feb & Mar & Apr & May & Jun & Jul & Aug & Sep & Oct & Nov & Dec & $>1$ & $0.5-1.0$. & $<0.5$ & $<1$ \\
\hline 2008 & - & - & - & - & - & - & - & - & - & - & - & - & - & 0 & 0 & 0 \\
2009 & - & - & - & - & - & - & - & - & - & 3.69 & 0.21 & 0.30 & 1 & 0 & 2 & 0 \\
2010 & 0.71 & 0.90 & 0.82 & 0.33 & 0.67 & 0.32 & 0.43 & 0.60 & 0.49 & 0.26 & 0.82 & 0.97 & - & 7 & 5 & 12 \\
2011 & 0.46 & 0.59 & 0.96 & 0.50 & 0.87 & 1.05 & 0.21 & 0.64 & 0.33 & 0.66 & 0.69 & 1.07 & 2 & 7 & 3 & 10 \\
2012 & 0.25 & 0.40 & 0.67 & 0.79 & 0.26 & 0.25 & 0.27 & 1.15 & 0.48 & 1.03 & 1.37 & 0.53 & 3 & 3 & 6 & 9 \\
\hline
\end{tabular}

Table 27. Ratio Between Standard Deviation of Basis and Spot Prices of Chana

\begin{tabular}{cccccccccccccccccc}
\hline Year & Jan & Feb & Mar & Apr & May & Jun & Jul & Aug & Sep & Oct & Nov & Dec & $>1$ & $0.5-1.0$. & $<0.5$ & $<1$ \\
\hline 2008 & 2.17 & 0.26 & 0.89 & 0.46 & 1.13 & - & - & - & - & - & - & 0.56 & 2 & 1 & 2 & 3 \\
2009 & 0.57 & 0.95 & 1.14 & 0.72 & 0.75 & 0.85 & 0.53 & 1.09 & 1.86 & 0.62 & 1.13 & 1.92 & 5 & 7 & 0 & 7 \\
2010 & 0.63 & 0.56 & 0.79 & 0.60 & 1.41 & 0.44 & 0.64 & 1.83 & 1.28 & 0.87 & 0.70 & 1.25 & 4 & 7 & 1 & 8 \\
2011 & 0.51 & 0.80 & 0.89 & 1.52 & 0.34 & 0.42 & 0.44 & 0.40 & 0.37 & 0.66 & 0.48 & 0.51 & 1 & 5 & 6 & 11 \\
2012 & 0.52 & 0.34 & 0.83 & 0.61 & 0.53 & 0.72 & 0.39 & 1.16 & 0.36 & 0.44 & 1.34 & 0.64 & 2 & 6 & 4 & 10 \\
\hline
\end{tabular}


Table 28. Ratio Between Standard Deviation of Basis and Spot Prices of Guar Seed

\begin{tabular}{|c|c|c|c|c|c|c|c|c|c|c|c|c|c|c|c|c|}
\hline \multirow[b]{2}{*}{ Year } & \multirow[b]{2}{*}{ Jan } & \multirow[b]{2}{*}{ Feb } & \multirow[b]{2}{*}{ Mar } & \multirow[b]{2}{*}{ Apr } & \multirow[b]{2}{*}{ May } & \multirow[b]{2}{*}{ Jun } & \multirow[b]{2}{*}{ Jul } & \multirow[b]{2}{*}{ Aug } & \multirow[b]{2}{*}{ Sep } & \multirow[b]{2}{*}{ Oct } & \multirow[b]{2}{*}{ Nov } & \multirow[b]{2}{*}{ Dec } & \multicolumn{4}{|c|}{ No of Times } \\
\hline & & & & & & & & & & & & & $>1$ & $0.5-1.0$. & $<0.5$ & $<1$ \\
\hline 2008 & 1.41 & 0.69 & 1.70 & 0.50 & 0.99 & 0.73 & 0.80 & 0.54 & 0.58 & 0.23 & 0.90 & 0.68 & 2 & 9 & 1 & 10 \\
\hline 2009 & 1.16 & 0.58 & 1.21 & 0.35 & 0.68 & 1.00 & 0.29 & 0.58 & 0.70 & 0.23 & 0.62 & 0.57 & 3 & 6 & 3 & 9 \\
\hline 2010 & 0.23 & 0.39 & 0.59 & 0.54 & 0.55 & 0.39 & 0.48 & 0.25 & 0.48 & 0.58 & 0.29 & 0.79 & 0 & 5 & 7 & 12 \\
\hline 2011 & 0.38 & 0.18 & 0.33 & 0.14 & 0.50 & 0.46 & 0.32 & 0.52 & 0.26 & 0.81 & 0.74 & 0.37 & 0 & 4 & 8 & 12 \\
\hline 2012 & 0.19 & 0.12 & 0.12 & - & 0.42 & 0.04 & - & - & - & - & - & - & 0 & 0 & 5 & 5 \\
\hline
\end{tabular}

Table 29. Ratio Between Standard Deviation of Basis and Spot Prices of Mustard Seed

\begin{tabular}{cccccccccccccccccc}
\hline \multirow{2}{*}{ Year } & Jan & Feb & Mar & Apr & May & Jun & Jul & Aug & Sep & Oct & Nov & Dec & $>1$ & $0.5-1.0$. & $<0.5$ & $<1$ \\
\hline & 0.62 & 1.64 & 0.32 & 0.79 & 0.65 & 0.63 & 0.86 & 1.11 & 0.88 & 1.08 & 2.41 & 1.23 & 5 & 6 & 1 & 7 \\
2009 & 0.64 & 0.80 & 0.55 & 0.37 & 0.75 & 0.78 & 0.54 & 0.68 & 1.37 & 0.38 & 0.53 & 0.76 & 1 & 9 & 2 & 11 \\
2010 & 0.79 & 0.82 & 0.51 & 0.70 & 0.27 & 0.76 & 0.29 & 0.57 & 0.64 & 0.80 & 0.86 & 1.03 & 1 & 9 & 2 & 11 \\
2011 & 0.81 & 6.49 & 0.44 & 1.35 & 0.39 & 0.63 & 0.28 & 0.97 & 1.72 & 1.04 & 0.29 & 0.41 & 4 & 3 & 5 & 8 \\
2012 & 0.57 & 0.40 & 0.27 & 0.61 & 0.92 & 0.97 & 0.25 & 0.74 & 0.59 & 0.36 & 1.45 & 1.70 & 2 & 6 & 4 & 10 \\
\hline
\end{tabular}

Table 30. Ratio Between Standard Deviation of Basis and Spot Prices of Pepper

\begin{tabular}{|c|c|c|c|c|c|c|c|c|c|c|c|c|c|c|c|c|}
\hline \multirow[b]{2}{*}{ Year } & \multirow[b]{2}{*}{ Jan } & \multirow[b]{2}{*}{ Feb } & \multirow[b]{2}{*}{ Mar } & \multirow[b]{2}{*}{ Apr } & \multirow[b]{2}{*}{ May } & \multirow[b]{2}{*}{ Jun } & \multirow[b]{2}{*}{ Jul } & \multirow[b]{2}{*}{ Aug } & \multirow[b]{2}{*}{ Sep } & \multirow[b]{2}{*}{ Oct } & \multirow[b]{2}{*}{ Nov } & \multirow[b]{2}{*}{ Dec } & \multicolumn{4}{|c|}{ No of Times } \\
\hline & & & & & & & & & & & & & $>1$ & $0.5-1.0$ & $<0.5$ & $<1$ \\
\hline 2008 & 0.39 & 0.74 & 0.62 & 1.02 & 0.78 & 0.97 & 1.11 & 2.63 & 0.60 & 0.33 & 0.96 & 0.49 & 3 & 6 & 3 & 9 \\
\hline 2009 & 0.39 & 0.27 & 0.59 & 0.20 & 0.54 & 1.63 & 1.09 & 0.21 & 0.91 & 0.96 & 0.83 & 0.33 & 2 & 5 & 5 & 10 \\
\hline 2010 & 1.10 & 1.02 & 0.35 & 0.57 & 1.18 & 0.44 & 0.26 & 0.37 & 0.62 & 0.30 & 1.81 & 1.43 & 5 & 2 & 5 & 7 \\
\hline 2011 & 2.39 & 0.52 & 0.97 & 0.31 & 0.66 & 0.87 & 0.59 & 0.37 & 0.37 & 1.40 & 2.19 & 0.68 & 3 & 6 & 3 & 9 \\
\hline 2012 & 0.96 & 0.16 & 0.35 & 1.24 & 0.40 & 0.60 & 0.73 & 2.33 & 0.97 & 4.05 & 0.24 & 1.34 & 4 & 4 & 4 & 8 \\
\hline
\end{tabular}

Table 31. Ratio Between Standard Deviation of Basis and Spot Prices of Potato

\begin{tabular}{|c|c|c|c|c|c|c|c|c|c|c|c|c|c|c|c|c|}
\hline \multirow[b]{2}{*}{ Year } & \multirow[b]{2}{*}{ Jan } & \multirow[b]{2}{*}{ Feb } & \multirow[b]{2}{*}{ Mar } & \multirow[b]{2}{*}{ Apr } & \multirow[b]{2}{*}{ May } & \multirow[b]{2}{*}{ Jun } & \multirow[b]{2}{*}{ Jul } & \multirow[b]{2}{*}{ Aug } & \multirow[b]{2}{*}{ Sep } & \multirow[b]{2}{*}{ Oct } & \multirow[b]{2}{*}{ Nov } & \multirow[b]{2}{*}{ Dec } & \multicolumn{4}{|c|}{ No of Times } \\
\hline & & & & & & & & & & & & & $>1$ & 0.5-1.0. & $<0.5$ & $<1$ \\
\hline 2008 & - & - & 0.22 & 0.91 & 0.63 & - & - & - & - & - & - & - & 0 & 2 & 1 & 3 \\
\hline 2009 & - & - & 0.76 & 0.84 & 0.96 & 0.46 & 3.24 & 0.71 & 0.39 & - & - & - & 1 & 4 & 2 & 6 \\
\hline 2010 & 0.10 & 0.76 & 0.39 & 0.67 & 0.76 & 3.36 & 0.24 & 0.82 & 0.88 & 1.71 & 0.57 & 0.15 & 2 & 6 & 4 & 10 \\
\hline 2011 & 0.20 & 0.10 & 0.88 & 2.98 & 2.43 & 2.81 & 2.37 & 1.92 & 8.51 & 3.31 & 1.10 & - & 8 & 1 & 2 & 3 \\
\hline 2012 & 0.09 & 0.15 & 1.71 & 1.11 & 4.24 & 5.63 & 0.43 & 1.47 & 0.67 & 0.94 & 1.15 & 0.96 & 6 & 3 & 3 & 6 \\
\hline
\end{tabular}

Table 32. Ratio Between Standard Deviation of Basis and Spot Prices of Soyabean

\begin{tabular}{ccccccccccccccccc}
\hline Year & Jan & Feb & Mar & Apr & May & Jun & Jul & Aug & Sep & Oct & Nov & Dec & $>1$ & $0.5-1.0$. & $<0.5$ & $<1$ \\
\hline 2008 & 0.28 & 0.32 & 0.48 & 0.24 & 0.18 & 0.54 & 2.33 & 0.42 & 0.39 & 0.28 & 0.15 & 0.18 & 1 & 1 & 10 & 11 \\
2009 & 0.22 & 0.94 & 0.38 & 0.30 & 1.20 & 0.18 & 0.59 & 0.39 & 0.38 & 0.30 & 0.51 & 0.20 & 1 & 3 & 8 & 11 \\
2010 & 0.37 & 0.41 & 0.86 & 0.97 & 0.44 & 0.27 & 0.47 & 0.91 & 0.31 & 0.66 & 0.54 & 0.47 & 0 & 5 & 7 & 12 \\
2011 & 0.34 & 0.35 & 0.88 & 0.51 & 0.58 & 0.72 & 1.06 & 0.22 & 0.27 & 0.68 & 0.24 & 1.47 & 2 & 5 & 5 & 10 \\
2012 & 0.53 & 0.49 & 0.30 & 0.54 & 0.35 & 0.22 & 5.59 & 0.57 & 0.28 & 0.72 & 0.51 & 0.53 & 1 & 6 & 5 & 11 \\
\hline
\end{tabular}


Future Trading in India and Commodity Price Risk Management: A Pragmatic Study

Table 33. Ratio Between Standard Deviation of Basis and Spot Prices of Aluminium

\begin{tabular}{ccccccccccccccccc}
\hline Year & Jan & Feb & Mar & Apr & May & Jun & Jul & Aug & Sep & Oct & Nov & Dec & $>1$ & $0.5-1.0$. & $<0.5$ & $<1$ \\
\hline 2008 & 2.15 & 0.92 & 1.69 & 1.17 & 2.74 & 1.95 & 2.65 & 1.12 & 0.84 & 3.53 & 1.85 & 1.49 & 10 & 2 & - & 2 \\
2009 & 0.89 & 1.43 & 1.30 & 1.37 & 2.89 & 2.68 & 1.93 & 2.51 & 2.33 & 0.84 & 0.79 & 3.13 & 9 & 3 & - & 3 \\
2010 & 2.13 & 1.51 & 0.94 & 1.77 & 2.41 & 1.17 & 0.85 & 1.85 & 1.21 & 1.74 & 3.16 & 1.90 & 10 & 2 & - & 2 \\
2011 & 1.95 & 2.97 & 1.27 & 2.55 & 1.28 & 2.08 & 1.97 & 2.67 & 1.05 & 2.43 & 1.38 & 1.51 & 12 & - & - & - \\
2012 & 1.55 & 1.07 & 0.73 & 1.09 & 1.31 & 0.89 & 1.24 & 1.06 & 1.50 & 1.19 & 2.09 & 1.30 & 10 & 2 & - & 2 \\
\hline
\end{tabular}

Table 34. Ratio Between Standard Deviation of Basis and Spot Prices of Copper

\begin{tabular}{ccccccccccccccccc}
\hline Year & Jan & Feb & Mar & Apr & May & Jun & Jul & Aug & Sep & 0ct & Nov & Dec & $>1$ & $0.5-1.0$. & $<0.5$ & $<1$ \\
\hline 2008 & 0.83 & 0.27 & 0.69 & 1.12 & 0.79 & 0.32 & 0.39 & 0.51 & 0.66 & 0.39 & 0.61 & 0.39 & 1 & 6 & 5 & 11 \\
2009 & 0.97 & 0.70 & 0.46 & 0.51 & 0.78 & 0.87 & 0.29 & 0.52 & 0.56 & 0.49 & 0.41 & 0.44 & - & 7 & 5 & 12 \\
2010 & 0.54 & 0.38 & 0.78 & 0.41 & 0.78 & 0.69 & 0.29 & 0.77 & 0.98 & 0.77 & 0.90 & 0.29 & - & 8 & 4 & 12 \\
2011 & 1.01 & 0.53 & 0.54 & 0.71 & 0.70 & 0.94 & 0.71 & 0.45 & 0.34 & 0.53 & 1.20 & 0.61 & 2 & 8 & 2 & 10 \\
2012 & 0.51 & 0.78 & 0.86 & 0.44 & 0.40 & 0.89 & 0.84 & 0.59 & 0.47 & 0.53 & 0.52 & 0.87 & - & 9 & 3 & 12 \\
\hline
\end{tabular}

Table 35. Ratio Between Standard Deviation of Basis and Spot Prices of Gold

\begin{tabular}{ccccccccccccccccc}
\hline Year & Jan & Feb & Mar & Apr & May & Jun & Jul & Aug & Sep & Oct & Nov & Dec & $>1$ & $0.5-1.0$. & $<0.5$ & $<1$ \\
\hline 2008 & 0.08 & 0.08 & 0.07 & 0.07 & 0.07 & 0.07 & 0.07 & 0.06 & 0.06 & 0.06 & 0.06 & 0.06 & - & - & 12 & 12 \\
2009 & 0.08 & 0.08 & 0.08 & 0.07 & 0.07 & 0.07 & 0.06 & 0.06 & 0.06 & 0.05 & 0.05 & 0.05 & - & - & 12 & 12 \\
2010 & 0.05 & 0.05 & 0.05 & 0.06 & 0.06 & 0.06 & 0.06 & 0.05 & 0.05 & 0.05 & 0.05 & 0.06 & - & - & 12 & 12 \\
2011 & 0.06 & 0.07 & 0.08 & 0.08 & 0.06 & 0.05 & 0.04 & 0.04 & 0.04 & 0.03 & 0.03 & 0.03 & - & - & 12 & 12 \\
2012 & 0.03 & 0.03 & 0.03 & 0.03 & 0.03 & 0.03 & 0.02 & 0.02 & 0.02 & 0.02 & 0.02 & 0.03 & - & - & 12 & 12 \\
\hline
\end{tabular}

Table 36. Ratio Between Standard Deviation of Basis and Spot Prices of Guar Silver

\begin{tabular}{ccccccccccccccccc}
\hline Year & Jan & Feb & Mar & Apr & May & Jun & Jul & Aug & Sep & Oct & Nov & Dec & $>1$ & $0.5-1.0$. & $<0.5$ & $<1$ \\
\hline 2008 & 0.74 & 0.40 & 0.90 & 0.59 & 0.34 & 0.66 & 0.71 & 0.21 & 0.37 & 0.37 & 0.36 & 0.49 & - & 5 & 7 & 12 \\
2009 & 0.40 & 0.27 & 0.91 & 0.49 & 0.68 & 0.78 & 0.41 & 0.60 & 0.49 & 0.65 & 0.34 & 0.31 & - & 5 & 7 & 12 \\
2010 & 0.32 & 0.55 & 0.68 & 0.68 & 0.47 & 0.75 & 0.98 & 0.49 & 0.26 & 0.39 & 0.66 & 0.44 & - & 6 & 6 & 12 \\
2011 & 0.38 & 0.18 & 0.33 & 0.14 & 0.50 & 0.46 & 0.32 & 0.52 & 0.26 & 0.81 & 0.74 & 0.37 & - & 4 & 8 & 12 \\
2012 & 0.39 & 0.74 & 0.73 & 1.42 & 0.53 & 0.66 & 1.11 & 0.21 & 1.31 & 0.60 & 0.36 & 0.43 & 3 & 5 & 4 & 9 \\
\hline
\end{tabular}

Table 37. Ratio Between Standard Deviation of Basis and Spot Prices of Nickel

\begin{tabular}{ccccccccccccccccc}
\hline Year & Jan & Feb & Mar & Apr & May & Jun & Jul & Aug & Sep & Oct & Nov & Dec & $>1$ & $0.5-1.0$. & $<0.5$ & $<1$ \\
\hline 2008 & 0.67 & 0.25 & 0.46 & 0.71 & 0.26 & 0.40 & 0.23 & 0.20 & 0.25 & 0.29 & 0.67 & 0.62 & - & 4 & 8 & 12 \\
2009 & 0.43 & 0.24 & 0.48 & 0.30 & 0.44 & 0.39 & 0.34 & 0.63 & 0.46 & 0.67 & 0.31 & 0.20 & - & 2 & 10 & 12 \\
2010 & 0.55 & 0.17 & 0.33 & 0.49 & 0.42 & 0.47 & 0.26 & 0.44 & 0.41 & 0.53 & 0.39 & 0.48 & - & 2 & 10 & 12 \\
2011 & 0.30 & 0.70 & 0.31 & 0.52 & 0.38 & 0.49 & 0.44 & 0.47 & 0.30 & 0.59 & 0.78 & 0.40 & - & 4 & 8 & 12 \\
2012 & 0.37 & 0.44 & 0.50 & 0.80 & 0.86 & 0.64 & 0.29 & 0.32 & 0.22 & 0.22 & 0.29 & 0.64 & - & 4 & 8 & 12 \\
\hline
\end{tabular}


Table 38. Ratio Between Standard Deviation of Basis and Spot Prices of Zinc

\begin{tabular}{ccccccccccccccccc}
\hline Year & Jan & Feb & Mar & Apr & May & Jun & Jul & Aug & Sep & 0ct & Nov & Dec & $>1$ & $0.5-1.0$. & $<0.5$ & $<1$ \\
\hline 2008 & 0.44 & 0.37 & 0.27 & 0.73 & 0.31 & 0.63 & 0.44 & 0.34 & 0.64 & 0.29 & 0.48 & 0.33 & - & 3 & 9 & 12 \\
2009 & 0.55 & 0.70 & 0.39 & 0.33 & 0.49 & 0.59 & 0.32 & 0.93 & 0.66 & 0.30 & 0.62 & 0.34 & - & 6 & 6 & 12 \\
2010 & 0.19 & 0.42 & 0.59 & 0.77 & 0.42 & 0.44 & 0.28 & 0.53 & 0.77 & 0.25 & 0.23 & 0.32 & - & 4 & 8 & 12 \\
2011 & 0.39 & 0.75 & 0.33 & 0.34 & 0.43 & 0.57 & 0.31 & 0.47 & 0.44 & 0.58 & 0.33 & 0.23 & - & 3 & 9 & 12 \\
2012 & 0.41 & 0.40 & 0.81 & 0.39 & 0.56 & 0.49 & 0.62 & 0.52 & 0.27 & 0.29 & 0.26 & 0.52 & - & 5 & 7 & 12 \\
\hline
\end{tabular}

Table 39. Ratio Between Standard Deviation of Basis and Spot Prices of Crude Oil

\begin{tabular}{ccccccccccccccccc}
\hline Year & Jan & Feb & Mar & Apr & May & Jun & Jul & Aug & Sep & Oct & Nov & Dec & $>1$ & $0.5-1.0$. & $<0.5$ & $<1$ \\
\hline 2008 & 0.26 & 0.21 & 0.60 & 0.19 & 0.29 & 0.45 & 0.22 & 0.51 & 0.46 & 0.22 & 0.34 & 0.37 & - & 2 & 10 & 12 \\
2009 & 0.44 & 0.50 & 0.40 & 0.71 & 0.33 & 0.64 & 0.41 & 0.72 & 0.57 & 0.34 & 0.79 & 0.27 & - & 6 & 6 & 12 \\
2010 & 0.15 & 0.32 & 1.00 & 0.59 & 0.27 & 0.51 & 0.30 & 0.24 & 0.72 & 1.00 & 0.71 & 0.53 & 1 & 6 & 5 & 11 \\
2011 & 0.87 & 0.57 & 0.63 & 0.53 & 0.48 & 0.43 & 0.86 & 0.51 & 0.80 & 0.31 & 0.35 & 0.71 & - & 8 & 4 & 12 \\
2012 & 0.29 & 0.27 & 0.76 & 0.46 & 0.34 & 0.88 & 0.48 & 0.40 & 0.28 & 0.67 & 0.70 & 0.35 & - & 4 & 8 & 12 \\
\hline
\end{tabular}

Table 40. Ratio Between Standard Deviation of Basis and Spot Prices of Natural Gas

\begin{tabular}{ccccccccccccccccc}
\hline Year & Jan & Feb & Mar & Apr & May & Jun & Jul & Aug & Sep & Oct & Nov & Dec & $>1$ & $0.5-1.0$. & $<0.5$ & $<1$ \\
\hline 2008 & 0.79 & 0.28 & 0.56 & 0.30 & 0.33 & 0.43 & 0.16 & 0.68 & 0.83 & 0.68 & 0.92 & 0.43 & - & 6 & 6 & 12 \\
2009 & 0.21 & 0.50 & 0.64 & 0.59 & 0.37 & 0.73 & 0.65 & 0.33 & 0.34 & 0.80 & 0.45 & 0.34 & - & 5 & 7 & 12 \\
2010 & 0.73 & 0.38 & 0.23 & 1.05 & 0.36 & 0.60 & 0.74 & 0.30 & 0.88 & 0.38 & 0.35 & 0.56 & 1 & 5 & 6 & 11 \\
2011 & 0.67 & 0.27 & 0.32 & 0.42 & 0.48 & 0.34 & 0.45 & 0.94 & 0.99 & 0.55 & 0.41 & 0.35 & - & 4 & 8 & 12 \\
2012 & 0.31 & 0.97 & 0.59 & 0.37 & 0.40 & 0.40 & 0.56 & 0.46 & 0.47 & 0.89 & 0.50 & 0.59 & - & 5 & 7 & 12 \\
\hline
\end{tabular}

$25 \%$ respectively. This indicates that guar seed is slightly less risky compared to mustard seed which is highly riskier. Further, in both the commodities the basis risk is getting reduced over the years slowly but surely.

- Even in the case of pepper and potato in less than $40 \%$ of the cases ratio between basis and spot price variations is within the benchmark ratio which shows that even these commodities carry relatively higher basis risk.

- In the case of Soya bean, however around $60 \%$ of the instance the ratio was below the benchmark and even the percentage of cases above ratio 1.00 is very less which indicates trading in soya bean is less risky than others. However, the trend indicates that over the years, basis risk is on the raise for soya bean.

- In case of metals like Gold, Nickel, Silver and Zinc, the contracts are less risky as they have higher percentage of cases wherein the ratio was below the bench mark and in least or none of the cases the ratio has exceeded ratio 1 . Specifically, in case of Gold all ratios were within the benchmark.

- On the other hand, while ratio for copper basis variability to spot prices was above the permitted level in $60 \%$ of the cases. However, the ratio exceeded only 3 times in 60 cases which we can take as contract with moderate risk requiring careful planning.

- Of all Aluminium contracts exhibited highest basis risk with none of the cases its ratio matching the bench mark and ratio surpassing 1.00 levels in 51 out of 60 months. This means that Aluminium contracts carry highest basis risk for the investors.

- In case of crude oil and natural gas contracts the ratio between standard deviation of basis and spot prices indicates that more than $55 \%$ of the ratio was qualifying the benchmark and the contracts are comparatively less risky in terms of basis risk. 


\subsubsection{Summary of Results}

The ratio analysis of standard deviations of monthwise daily futures and spot prices showed that Pepper, Soya bean, Copper, Gold, Nickel, Silver, Zinc, Crude oil and Natural gas exhibited higher levels of efficiency in terms of information utilisation and in case of metals and energy the efficiency levels were highest. Similarly, Castor seed, Guar seed, Mustard seed, and Potato indicated moderate levels of efficiency contrary to Barley, Chana which was found to be highly inefficient.

Basis risk analysis shows that metals (except Aluminium and Copper) and energy commodities are efficient and risk free. On the other hand Castor seed, Guar seed, Mustard seed, Pepper, Potato and Copper indicated moderate level basis risk against Barley, Soya bean and Aluminium which showed highest levels of basis risk.

\subsection{Constraints and Challenges}

Wrong understanding of the derivative markets as the playground for speculation and responsible for inflationary trends is the main reason for the underdevelopment of the industry in the past. Discussed below are few limitations that could be found in Indian derivatives markets.

- Hawala markets which are often localized operate with very low transaction costs and hence attract large number of speculators and small hedgers. Dispersed, fragmented and being in its stage of infancy, the Indian commodity markets are to achieve minimum critical liquidity in many thin commodities. In thin markets, the transactions of individual hedgers have significant price effects and result in substantial 'transaction costs'.

- The frequent interferences from the government and long period of prohibition have resulted in driving a part of the trade underground, with a large number of participants shifting to other professions, including securities market. These markets in India remained isolated from rapid advances in the systems of brokerages, market designs, trading, clearing, settlement, and governance of exchanges since 1970s, when derivatives were introduced in Western markets.
- The physical or spot markets in India are small and fragmented and it is difficult to operate national level exchanges on fragmented localized cash markets.

- Restriction on freedom to diversify for regional exchanges and need to seek fresh recognition each time to add new commodities are hurting their activities.

- Differential tax structures both in octroi and stamp duty by states remain unresolved and it is believed that charge of high stamp duties (for non-delivery transactions) by state governments are leading to illegal hawala markets.

- Apart from physical/infrastructural limitations such as limited online trading, online surveillance and monitoring, the non-availability of full proof legal system of contracts, particularly relating to the warehouse receipt system, etc., are seriously constraining the derivatives market.

- Smaller land holdings, excessive dependence on monsoon, poor agricultural inputs and technology, lack of rural infrastructure (warehousing, grading/ sorting facilities, access roads to markets), poor flow of price and market information all combine to translate to unsteady output, sub-standard quality and fluctuating farm-gate prices.

- The operations of cartels, who are also the members of few national exchanges, are resulting in unforeseen squeeze, cornering and monopolising stocks as well as dumping of stocks for price rigging both ways, up and down, and affecting the interests of real stakeholders. Non-delivery of material or receipt of money has put some of the stakeholders in such difficulty that they could not pay the growers the money for their produce. Therefore, a good section in the trade, feel that the operators at the national level commodity exchanges require to be controlled and certain commodities such as pepper, guar seed, guar gum, chillies, cumin, turmeric etc. is restricted to the respective regional commodity specific exchanges only.

- The membership of commodity exchanges in India is found to be limited and closed in nature. Non-participation of genuine hedgers is affecting the derivatives trading negatively. Concentration of trading in only few selected commodities and smaller turnovers in others are leading the corporate houses to look for offshore markets. 
- The systems of administered price mechanism, Minimum Support Pricing and frequent market interventions by the government are making the mockery of basic principle of market forces determining prices commodities and are found to contradict the development of commodity exchanges in India.

- Another main issue hindering the real growth of commodity derivatives is the lack of awareness of the role and technique of derivative trading among the potential beneficiaries which is hindering the growth of the market. Small size of the farmers and inadequate access to credit has also dampened the development of this market in India.

- The fundamentals that drive commodity markets are totally different from the factors affecting equity markets. Further, point that futures market is a success story in western world cannot be reckoned for assured success in India. There is a need to conduct in-depth studies to understand the fundamental differences in market structures, production, demand and supply conditions, stakeholders' interest, cost and benefit of implementation etc. Such studies will help in better understanding of dynamics, thereby better policy decisions and better results for all the stakeholders.

- Forward Markets Commission exercises delegated powers and have very limited authority to function in India. As the responsibilities creating conducive environment with necessary policies and infrastructure rests with the commission, it needs to garner more resources-human, financial, infrastructural etc. The FMC to be given more regulatory powers to take stringent actions against the culprits behind the wrong state of affairs in few selected markets as well as take necessary steps for the revival of regional commodity exchanges across India.

\section{Conclusion}

The present study aims to study the operational efficiency of commodity derivatives in India in price risk management. The present study is an analytical study based on secondary data. Secondary data consisting of daily spot and nearest month future prices were taken for selected agricultural, metal and energy commodities traded in India derivatives market. For markets to be efficient there has to be co-movement between both the spot and future prices. In the finance literature, a pre-condition for market efficiency is the convergence of both future and spot prices across the market spectrum. The co-integration analysis between the spot and futures prices on all 16 selected commodities indicated the existence of long-run relationship between the prices. The price volatility analysis between spot and futures prices as well as between basis and spot prices indicated highest level of efficiency in all metal (except Aluminium) and energy contracts. On the other hand agricultural commodities barley, chana and soyabean indicated higher levels of inefficiency than others.

In view of the promising prospects that these markets hold, following issues need to be addressed. Concerted efforts have to be made to bring the traditional players to the formal market in order to achieve minimum critical liquidity, sufficient breadth and depth, and provide relatively less expensive exit route. There is a need to strengthen the input delivery system, expansion of irrigation facilities, ensure timely and adequate credit delivery, educate farmers about agronomy and enable them to follow pre and post harvest scientific practices. Besides, putting in place adequate rural infrastructure for warehousing and dissemination of price and market information to farmers warrants renewed thrust.

\section{References}

Asche F., \& Guttormsen (2002). Lead lag relationships between futures and spot prices. Working Paper, Institute for Research in Economics and Business Administration, Bergen.

Beck, E.S.(1994). Co-integration and market efficiency in commodities futures markets. Applied Economics, 26, 249-257.

Chowdhury, A.R. (1991). Futures market efficiency: evidence from co-integration tests. The Journal of Futures Markets, 11, 577-589.

Cox, C.C. (1976). Futures trading and market information. Journal of Political Economy, 84, 1215-1237.

Dimitris, F. K., \& Aristeidis, G. S. (2004). Testing efficiency of the copper futures market. New Evidence from London Metal Exchange. Global Business and Economic Review, Anthology 2004, 261-271.

Figlewski, S. (1981). Futures trading and volatility in the GNMA Market. Journal of Finance, 36, 445-456. 
Garbade K.D., \& Silber W.L. (1983). Price movements and price discovery in futures and cash markets, Review of Economics and Statistics, 65, 289-297.

Lokare, S.M. (2007). Commodity derivatives and price risk management: An Empirical Anecdote from India. Reserve Bank of India Occasional Papers, 28, 27-77.

Morgan C.W. (1999). Futures markets and spot price volatility: a case study. Journal of Agricultural Economics, 50, 247-257.

Naik G, \& Jain S.K. (2002). Indian agricultural commodity futures markets: a performance survey. Economic and Political Weekly. 37, 3161-3173.

Netz, J.S. (1995). The effect of futures markets and corners on storage and spot price volatility. American Journal of Agricultural Economics, 77, 182-193.

Pantisa, P., \& Piyamas, C. (2010). Price discovery in the Indian Gold Futures Market. Journal of Economics and Finance, 2010, 455-467.

Turnovsky, S.J., \& Campbell, R.B. (1985). The stabilising and welfare properties of futures markets: a simulation approach. International Economic Review, 26, 277-303.

\section{About the Author(s)}

Dr. Nirmala K Reddy is a Professor in the Department of Commerce, Bangalore University, India and holds the degrees of M.Com. and Ph.D. Her area of specialization is Banking with focus on Derivatives. She has published papers in journals and conferences at international and national level. She can be contacted at: knirmalareddy@rediffmail.com

Chandra Shekar B. M. is a Research Scholar in the Department of Commerce, Bangalore University, India. He can be contacted at:chandrashekar.bm243@ gmail.com

Munilakshmi R. is a Research Scholar in the Department of Commerce, Bangalore University. She can be contacted at: mlakshmi15@gmail.com 